\title{
Limits to the representation capacity of imaging in random media
}

\author{
Aniceto Belmonte \\ Technical University of Catalonia, BarcelonaTech, Department of Signal Theory and Communications, \\ 08034 Barcelona, Spain (belmonte@tsc.upc.edu) \\ Received May 6, 2013; revised July 10, 2013; accepted July 11, 2013; \\ posted July 11, 2013 (Doc. ID 190068); published August 5, 2013
}

\begin{abstract}
The information capacity of an image in the atmosphere, ocean, or biological media does not grow indefinitely with increasing light power but has well defined limits. Here, the exact effects of the propagation of light in random inhomogeneous media are elucidated and upper bounds to the capacity of image pixels to represent a corresponding point in the object are described. (c) 2013 Optical Society of America

OCIS codes: (110.0115) Imaging through turbulent media; (110.3055) Information theoretical analysis; (010.0010) Atmospheric and oceanic optics; (170.3880) Medical and biological imaging.

http://dx.doi.org/10.1364/OL.38.002941
\end{abstract}

Optical systems have the capacity to represent physical objects by a certain pattern of received photons. Without considering the properties of the objects to be represented, information theory provides a framework where the limits to the capacity of a specific image system, or how much information can be extracted from a population of photons about the identity of the entity that is being represented, can be quantified. However, the nature of the propagation of light in random inhomogeneous media has made these limits difficult to elucidate and confines information-theoretic approaches to considering representational capacity in homogeneous media. Here, the precise effects of random media, those exhibiting great complexity and variability, are elucidated and upper bounds to the capacity of an image in the atmosphere, ocean, or biological media are described. The ideas presented here affect qualitatively the information capacity of optical applications, such as environmental spectroscopy or biomedical imaging, and may have broader implications for nonoptical wave applications ranging from neutron radiography to acoustic tomography to seismic imaging.

In this Letter we consider the role of random media in the theory of information content of imaging for direct (incoherent) detection and heterodyne (coherent) detection optical receivers. Although many imaging applications consider incoherent signals that are proportional to the received optical power, the most advanced coherent imaging techniques are based on the recovery of the full image field, which contains both amplitude and phase information. We use a generalized treatment of imaging, where any direct comparison of the representational capacity in the two detection systems must be in terms of the same observable quantity, the number of received photons. Although there are properties of images formed with coherent detection that should be mentioned in any comparison with incoherent images, the complex ways that the random media affects the amplitude $A$ and the phase $\phi$ of the optical fields will result in light fading and low resolution, influencing the information content of images in both detection systems.

As the down-converted heterodyne power is maximized when the spatial field of the received signal matches that of the local oscillator, any mismatch between the phases of the two fields is of the utmost importance and will result in a degradation of the available power, i.e., fading. Direct detection imaging is not sensitive to the phase of the optical fields and only wavefront amplitude fluctuations increase the intensity of fading. Also, in both direct and heterodyne detection imaging, random media can severely distort the spatial frequency response of the optical system and degrade its lateral optical resolution. In a perfect diffraction-limited optical lens system, the presence of a point source object yields an ideal diffraction point in the image plane. The aberration effect of random media keeps the point image apart from ideal and limits the practical optical resolution and total number of pixels of the image.

The classical theory of information [1] was developed originally in the context of linear channels with additive noise. Considering an imaging system as a communication channel allows it to be characterized in terms of Shannon's information theory ideas and to quantify statistically the information content of images. For an additive white Gaussian noise (AWGN) image, the classical capacity formula for an imaging system [2] with average power constraint $P$ and noise power spectral density $N_{0} / 2$, is given by the Shannon limit, $C=$ $B N_{F} \log _{2}\left(1+\gamma_{0}\right)$. Here, the spectral bandwidth $B$, which has units of $\mathrm{Hz}$, and the number of spatial degrees of freedom $N_{F}$ in the imaging system [3], or pixels, multiply the pixel spectral efficiency, $\log _{2}\left(1+\gamma_{0}\right)$, which has units of bits $/ \mathrm{s} / \mathrm{Hz} /$ pixel, and where $\gamma_{0}=P / N_{0} B$ is the SNR per unit bandwidth $B$. The SNR $\gamma_{0}$ for a quantum or shot-noise limited optical signal can be interpreted as the detected number of photons (photocounts) per pixel when $1 / B$ is the image integration time. Note that, for sufficient large photon numbers, the shot noise distribution approaches a normal distribution, typically making shot noise in actual observations indistinguishable from true AWGN, except when the elementary photons are so few that they are individually observed. Classical treatments of the problem are based primarily on the shot noise encountered in optical field detection, which leads to the conclusion that arbitrarily high representational capacities of imaging $C$ are possible.

Evaluating the performance of an imaging system in the presence of a random medium is generally more 
difficult because of the complex way the medium, whose basic properties are random functions of space and time, affects the amplitude and the phase of the received optical field. In imaging systems over turbulent media, we must consider fading signals [4], which are a class of optical signals with multiplicative noise $\alpha$. We contemplate imaging systems in the presence of multiplicative noise from turbulent media and AWGN. In the fading image with average power constraint $P$ and noise power spectral density $N_{0} / 2$, we let $\alpha^{2}$ denote the random power fading and $\left(P / N_{0} B\right) \alpha^{2}=\gamma_{0} \alpha^{2}$ denote the instantaneous SNR per pixel. Conditional upon a realization of the random medium described by $\alpha$, this is an image with an instantaneously received SNR per pixel of $\gamma=\gamma_{0} \alpha^{2}$, and the pixel spectral efficiency is given by $\log _{2}(1+\gamma)$. Note that $\gamma$ is a function of the random power fading $\alpha^{2}$ caused by amplitude and phase fluctuations and is therefore random. Hence, amplitude and phase fluctuations, and the corresponding fading SNR $\gamma$, need to be characterized.

Fading models, for both coherent and incoherent detection, consider a Kolmogorov statistical description of random inhomogeneous or turbulent media. Here, Gaussian $\log$-amplitude $\chi=\log A$ and phase $\phi$ fluctuations are characterized by their respective statistical variances, $\sigma_{\chi}^{2}=1 / 4 \log _{e}\left(1+\sigma_{\beta}^{2}\right)$ and $\sigma_{\phi}^{2}=1.0299\left(D / r_{0}\right)^{5 / 3}$. The intensity variance $\sigma_{\beta}^{2}$ is often referred to as the scintillation index. The aperture diameter $D$ of the optical lens system is normalized by the wavefront coherent diameter $r_{0}$, which describes the spatial correlation of phase fluctuations in the lens pupil plane.

The impact of media-induced phase and amplitude fluctuations on the SNR $\gamma$ per pixel in optical systems using coherent detection can be described by a noncentral chisquare probability distribution function with two degrees of freedom []․ The distribution can be integrated to obtain the corresponding cumulative distribution function

$$
F_{\gamma}(\gamma)=1-Q_{1}(\sqrt{2 r}, \sqrt{2(1+r) r \gamma / \bar{\gamma}}),
$$

where $Q_{1}$ is the first-order Marcum $Q$-function. The model leading to this distribution is based on the observation that the coherent signal can be characterized as the sum of many contributions from different wavefront coherent regions within the aperture, which is the cause of speckle [6]. The average SNR (or average detected photocounts per pixel) $\bar{\gamma}=\gamma_{0} \bar{\alpha}^{2}$ is expressed in terms of the fading mean-square value $\bar{\alpha}^{2}$ that depends on the inverse of the field coherent areas $N \approx\left(D / r_{0}\right)^{2}$ and affects the fading measurement. In this model, the signal is expressed as the sum of a constant (coherent) term and a random (incoherent) residual halo. The SNR, $r=\alpha_{0}^{2} / 2 \sigma_{\alpha}^{2}$, of the speckle pattern is a measure of the strength of the coherent component, $\alpha_{0}^{2}=\exp \left(-\sigma_{\chi}^{2}\right) \exp \left(-\sigma_{\phi}^{2}\right)$, relative to the residual halo, $2 \sigma_{\alpha}^{2}=\left(1-\alpha_{0}^{2}\right) / N$.

With incoherent detection there is a general consensus [7] that fading SNR $\gamma$ can be modeled by a log-normal distribution,

$$
F_{\gamma}(\gamma)=Q\left(-\log _{e}\left(\gamma / \gamma_{0}\right) / \sigma_{\beta}+\sigma_{\beta} / 2\right),
$$

where $Q$ is the Gaussian $Q$-function. Now, only amplitude fluctuations on the optical signals are relevant and power fading, $\alpha^{2}=\exp (2 \chi)$, or scintillation is characterized by the log-amplitude variance $\sigma_{\chi}^{2}$ through the scintillation index, $\sigma_{\beta}^{2}=\exp \left(4 \sigma_{\chi}^{2}\right)-1$.

We investigate the usefulness of various pixel representational capacity definitions (see Table 1), namely ergodic capacity, where it is assumed that the pixel transitions over all the fading states, and outage capacity, where the pixel accepts a specified outage (fade) probability. The exact effects of fading are described by replacing the SNR per pixel $\gamma_{0}$ in the Shannon's capacity limit $\log _{2}\left(1+\gamma_{0}\right)$ by the SNR parameter describing ergodic, outage, and zero-outage spectral efficiency upper bounds.

Ergodic capacity is used if the image integration time $1 / B$ spans many temporal fading states, i.e., $B \ll F$. Here, the rate $F$ at which the fading fluctuates will be dictated by the random medium dynamic properties and is generally low. For example, for atmospheric optical signal fluctuations $F$ is no higher than $1 \mathrm{kHz}$ and can be as small as a few tens of Hz. In this ergodic scenario, image integration takes place over all fading states, and allows the capacity to be expressed as an average over many independent fades of the random media and the ergodic capacity is the expectation $E$ with respect to the fadings of the instantaneous capacity, $B N_{F} \log _{2}(1+\gamma)$. Considering that the gain from the times when the signal fades are shallow cannot compensate for the loss for when signal fades are deep, because the pixel spectral efficiency term $\log _{2}(1+\gamma)$ is a concave function, Jensen's inequality provides an upper bound for the ergodic capacity, $E(C) \leq B N_{F} \log _{2}(1+\bar{\gamma})$. Ergodicity makes certain that the time-averaged SNR, $\bar{\gamma}=\gamma_{0} \bar{\alpha}^{2}$, converges to the same limit for all realizations of the fading process.

The expected ergodic pixel spectral efficiency for a heterodyne imaging system becomes upper bounded by $\log _{2}\left[1+\gamma_{0}\left(r_{0} / D\right)^{2}\right]$, reaching the Shannon spectral efficiency limit, $\log _{2}\left(1+\gamma_{0}\right)$, only when the lens aperture is smaller than one wavefront coherence diameter $r_{0}$. Two coherent regions within the aperture are already the cause of speckle [ $\underline{6}$ ] and introduce a SNR fading penalty

Table 1. We Investigate Various Pixel Representational Capacity Definitions of Imaging in Random Media for Direct and Heterodyne Detection Optical Receivers

\begin{tabular}{lcc}
\hline & Heterodyne & Direct \\
\hline Ergodic & $\bar{\gamma}=\gamma_{0}\left(r_{0} / D\right)^{2}$ & $\bar{\gamma}=\gamma_{0} \exp \left(-2 \sigma_{\chi}^{2}\right)$ \\
Outage & $\gamma_{\varepsilon} \leq \bar{\gamma} / 2(1+r)\left[\sqrt{-2 \log _{e}(1-\varepsilon)}+\sqrt{2 r}\right]^{2}$ & $\gamma_{\varepsilon} \leq \gamma_{0} \exp \left[-\sigma_{\beta}^{2} / 2+\sqrt{\left.2 \sigma_{\beta}^{2} \log (2 \varepsilon)\right] ;}\right.$ \\
Zero-outage & $\gamma_{\varepsilon \rightarrow 0} \leq \gamma_{0} \exp \left(-\sigma_{\chi}^{2}\right) \exp \left(-\sigma_{\phi}^{2}\right)$ & $\gamma_{\varepsilon \rightarrow 0} \leq \gamma_{0} \exp \left(-\sigma_{\beta}^{2} / 2\right)$ \\
\hline
\end{tabular}


factor of 2 (that is, $3 \mathrm{~dB}$ ) with respect to the AWGN Shannon spectral efficiency. When the number of contributions to the optical signal from different wavefront coherent regions within the aperture is larger, the ergodic bounds decrease and the image representational capacity decreases.

If direct detection imaging is considered, Jensen's bound to the pixel spectral efficiency is limited only by scintillation and becomes $\log _{2}\left[1+\gamma_{0} \exp \left(-2 \sigma_{\chi}^{2}\right)\right]$. A $3 \mathrm{~dB}$ SNR fading penalty is introduced by a log-amplitude variance of $\sigma_{\chi}^{2}=1 / 3$.

Ergodic capacity assumes that the signal fading evolves through all possible fading states as the basic properties of the propagation media changes randomly with time, and thus might not be very useful in practice for image systems with fixed integration time constraints. A very long integration time is required for achievability of ergodic capacity, the length being dependent on the dynamics of the fading process. Outage capacity is used where the instantaneous SNR $\gamma$ can be assumed to be constant during the image integration time. In particular, the integration time $1 / B$ must be smaller than the coherence time $1 / F$, defined to be the time over which the image is significantly correlated. When delay constraints prevent averaging over deep and shallow fading states, and the image integration is restricted to just one coherence time, strictly speaking, the image system capacity is zero because there is a chance that the fading might be so severe that the instantaneous capacity is below any desired information rate level. In this case, a more appropriate measure of capacity is the probability that the image can support a desired information rate. Unlike the ergodic capacity scenario, schemes designed to achieve outage capacity allow for image errors. Hence, in deep fades these schemes allow the image information to be lost and a higher information rate than schemes achieving ergodic capacity may be thereby maintained.

Specifically, an appropriate measure of outage capacity is the probability that the imaging system can support a given rate $C_{\varepsilon}$, i.e., $p_{\text {out }}\left(C_{\varepsilon}\right)=\mathbb{P}\left\{B N_{F} \log _{2}(1+\gamma)<C_{\varepsilon}\right\}$. Here, the operator $\mathbb{P}\{A\}$; indicates the probability of an event $A$. Let $\gamma_{\varepsilon}$ denote the SNR that is required to support a rate $C_{\varepsilon}$. As the image capacity is monotonically increasing with received power for a given random media state, the probability of outage can be expressed in terms of SNR as $p_{\text {out }}\left(C_{\varepsilon}\right)=\mathbb{P}\left\{\gamma<\gamma_{\varepsilon}\right\}$. This result can be expressed in terms of the complementary cumulative distribution function of the SNR $\gamma$ as $p_{\text {out }}\left(C_{\varepsilon}\right)=$ $1-F_{\gamma}\left(\gamma_{\varepsilon}\right)$. From here, we can solve $p_{\text {out }}\left(C_{\varepsilon}\right)=\varepsilon$ to obtain the SNR $\gamma_{\varepsilon}$, producing a $\varepsilon$-outage probability of $F_{c}\left(\gamma_{\varepsilon}\right)=$ $1-\varepsilon$, i.e., $\gamma_{\varepsilon}=F_{\gamma}^{-1}(1-\varepsilon)$. Then, by definition, the $\varepsilon$-outage capacity becomes $C_{\varepsilon}=B N_{F} \log _{2}\left[1+F_{\gamma}^{-1}(1-\varepsilon)\right]$. It is clear that the outage capacity depends on the statistical distribution of SNR $\gamma$ through its $F_{\gamma}\left(\gamma_{\varepsilon}\right)$. For received SNRs $\gamma_{\varepsilon}$ below $F_{\gamma}^{-1}(1-\varepsilon)$, the optical signal cannot be successfully interpreted and the system outage results in a distorted image.

For heterodyne detection imaging we represent the first-order Marcum $Q$-function in Eq. (1) by a strict upper Chernoff bound, $Q_{1}(a, b) \leq \exp \left[-(b-\bar{a})^{2} / 2\right]$, allowing the reversal of $F_{\gamma}\left(\gamma_{\varepsilon}\right)$ to obtain the argument $\gamma_{\varepsilon}=F_{\gamma}^{-1}(1-\varepsilon)$ as follows:

$$
\gamma_{\varepsilon} \leq \bar{\gamma} / 2(1+r)\left[\sqrt{-2 \log _{e}(1-\varepsilon)}+\sqrt{2 r}\right]^{2} .
$$

The applicability of these results is shown by noting that when the signal coherent term is very strong $(r \rightarrow \infty)$, and there is no fading to be considered, the log-amplitude and phase variances become very small, $\gamma_{\varepsilon} \approx \gamma_{0}$, and the pixel spectral efficiency tends toward the Shannon limit $\log _{2}\left(1+\gamma_{\varepsilon}\right)$. On the other hand, we note that, in the regime where the number of contributing coherent areas is large, the coherent part of the signal is very weak relative to the residual halo $(r \rightarrow 0)$ and the normalized variance of the SNR reaches a maximum, as expected in fully developed speckle. Now, the pixel spectral efficiency is negligible and no significant information can be extracted from the image. In the extreme case of requiring zero-outage probability, $\varepsilon \rightarrow 0$, the outage SNR [Eq. (3)] is bound by $\gamma_{\varepsilon \rightarrow 0} \leq \gamma_{0} \exp \left(-\sigma_{\chi}^{2}\right) \exp \left(-\sigma_{\phi}^{2}\right)$ and the outage capacity then becomes the zero-outage capacity. This can be interpreted to mean that the image data is always correctly decoded, resulting in a received pixel spectral efficiency of $\log _{2}\left[1+\gamma_{0} \exp \left(-\sigma_{\chi}^{2}\right) \exp \left(-\sigma_{\phi}^{2}\right)\right]$. Being concerned with the outage (fading) capacity, we use the zero-outage capacity as a metric for the maximum achievable rate of fading imaging systems with a zero-outage probability. When outages are unavoidable, the zero-outage capacity is zero. In a standard situation, for example when the partially developed speckle produced by two coherent regions within the aperture is considered along with a log-amplitude variance of $\sigma_{\chi}^{2}=1 / 3$, a large SNR fading penalty factor of 36 (almost $16 \mathrm{~dB}$ ) with respect to the pixel Shannon spectral efficiency should be expected and the image representational capacity would be very small. In most heterodyne detection applications, media-induced wavefront distortion becomes so severe that it would be unrealistic to require zero-outage probability for the imaging system.

For direct detection imaging, using the upper Chernoff bound, $Q(a) \leq \exp \left[-a^{2} / 2\right]$, to the Gaussian $Q$-function in Eq. (2), it becomes

$$
\gamma_{\varepsilon} \leq \gamma_{0} \exp \left[-\sigma_{\beta}^{2} / 2+\sqrt{2 \sigma_{\beta}^{2} \log _{e}(2 \varepsilon)}\right] .
$$

In this case, the zero-outage SNR $\gamma_{\varepsilon \rightarrow 0} \leq \gamma_{0} \exp \left(-\sigma_{\beta}^{2} / 2\right)$ results in the delay-limited pixel spectral efficiency bound, $\log _{2}\left[1+\gamma_{0} \exp \left(-\sigma_{\beta}^{2} / 2\right)\right]$, and the representational capacity of the image decreases when the scintillation index $\sigma_{\beta}^{2}$ increases. Now, with respect to the pixel Shannon efficiency, a log-amplitude variance of $\sigma_{\chi}^{2}=$ $1 / 3$ introduces a SNR fading penalty factor of 4 (that is, $6 \mathrm{~dB}$ ). As opposed to using heterodyne systems, many imaging applications using direct detection may support zero-outage probabilities.

The behavior of the pixel spectral efficiency is graphically illustrated in Figs. 1 and 2 in the slow (ergodic) and fast (outage) image integration-time scenarios, when heterodyne or direct detection receivers are considered. It is clear from Fig. 1 that, for a given probability of outage, there is a significant gain in the achievable spectral efficiency when utilizing the direct detection over the 


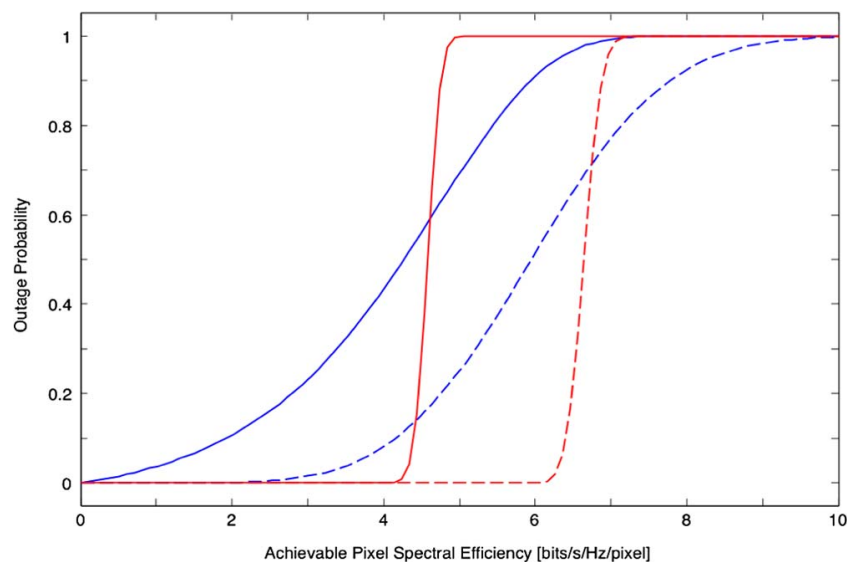

Fig. 1. Upper bounds to the outage pixel spectral efficiency for imaging in a random medium. Probability of outage versus image capacity per unit bandwidth and pixel for heterodyne detection (solid lines) and direct detection (dashed lines) are considered using Eqs. (3) and (4), respectively. The trade-off between the outage probability and the maximum achievable pixel spectral efficiency is analyzed for both fast image integration time (in blue) and slow integration times (in red) leading to the ergodic limit (vertical lines). In all cases, we assume the number of photons per pixel $\gamma_{0}$ equals 100 (i.e., $20 \mathrm{~dB}$ SNR). Random-media effects on the phase wavefront are characterized by a moderate phase coherence length $r_{0}$ such that four coherent regions are within the aperture, i.e., $D / r_{0}=2$. Wavefront amplitude fluctuations are considered by assuming $\sigma_{\gamma}^{2}=1 / 3$.

heterodyne detection. For example, in the fast-integration case considered in the figure, if the imaging system is designed to meet an outage probability of $\varepsilon=1 / 10$, then the maximum information that can be reliably obtained from each pixel in the direct detection image is around



Fig. 2. Curves represent upper bounds to the outage and ergodic pixel spectral efficiency for imaging in a random media as a function of the lens aperture diameter normalized to the wavefront coherent diameter $r_{0}$. For heterodyne detection (solid lines) and direct detection (dashed lines), pixel spectral efficiency bounds are analyzed for both fast (in blue, lower dashed line and bottom 3 curves) and slow (in red, top curve and top dashed line) integration times leading to the outage and ergodic limits, respectively. Outage probability levels of $0,1 / 100$ and $1 / 10$ are considered. For the smallest aperture considered, we assume $\gamma_{0}$ is equal to 100 photons-per-pixel. For any other aperture diameter, the value of $\gamma_{0}$ is proportional to $D^{2}$. Wavefront amplitude fluctuations consider $\sigma_{\gamma}^{2}=1 / 3$.
$4 \mathrm{bits} / \mathrm{s} / \mathrm{Hz} /$ pixel while, when utilizing a heterodyne detection system, it is less than 2 bits/s/Hz/pixel, which is an increase of over $50 \%$ in the achievable information rate. For slow integration times leading to ergodic capacities, pixel spectral efficiencies for direct and heterodyne detections differ by more than 2 bits/s/ $\mathrm{Hz} /$ pixel.

Figure 2 considers the effect of aperture diameter on the pixel spectral efficiency as a function of the normalized aperture $D / r_{0}$ for a constant phase coherence length $r_{0}$. If heterodyne detection is considered, zero-outage $(\varepsilon \rightarrow 0)$ capacity is impractical when the normalized aperture diameter is large and, in this regime, only schemes allowing for image errors can support nonzero pixel spectral efficiencies. Note the expanding capacity returns that are obtained as the accepted error $\varepsilon$ increases. However, as we increase the error, the outage spectral efficiency improves appreciably, and the greatest improvement is still obtained by going from fast to slow (ergodic) integration times. Even the largest apertures contemplated in the figure, which are a cause for intense speckle effects, reach a sizeable ergodic pixel spectral efficiency of $14 \mathrm{bits} / \mathrm{s} / \mathrm{Hz} / \mathrm{pixel}$. The existence of a limiting aperture diameter in heterodyne detection is apparent. With this aperture, the image capacity reaches a maximum level. When the aperture diameter is larger than the limiting value, phase distortion cannot be overcome by the increase in collected power, and we observe a saturation of the image capacity. As was noted before, higher outage pixel spectral efficiencies can also be obtained with large aperture diameters by using direct detection of images. However, when small aperture diameters are considered and speckle effects are diminished, heterodyne detection provides a higher degree of protection against scintillation than direct detection does. In this regime, heterodyne spectral efficiency can be almost 2 bits/s/Hz/pixel higher than the equivalent direct detection efficiency. In general, schemes using ergodic integration times and direct detection offer the closest pixel spectral efficiency to the Shannon limit.

In conclusion, fading affecting optical signals in random media can severely degrade information capacity and image representativeness. The fundamental contribution in the current work is the description of intuitive upper bounds (see Table 1) that can be used to describe the ultimate capacity of atmospheric, oceanic, or biological imaging.

This research was partially funded by the Spanish Department of Science and Innovation MICINN Grant No. TEC 2012-34799.

\section{References}

1. C. E. Shannon, Bell Syst. Tech. J. 27, 379 (1948).

2. J. A. O'Sullivan, R. E. Blahut, and D. L. Snyder, IEEE Trans. Inf. Theory 44, 2094 (1998).

3. G. T. di Francia, J. Opt. Soc. Am. 59, 799 (1969).

4. E. Biglieri, J. Proakis, and S. Shamai, IEEE Trans. Inf. Theory 44, 2619 (1998).

5. A. Belmonte and J. M. Kahn, Opt. Express 16, 14151 (2008).

6. J. W. Goodman, Speckle Phenomena in Optics. Theory and Applications (Ben Roberts, 2007).

7. L. C. Andrews and R. L. Phillips, Laser Beam Propagation Through Random Media, 2nd ed. (SPIE, 2005). 